\title{
Wearable EOG goggles: seamless sensing and context-awareness in everyday environments
}

\section{Journal Article}

Author(s):

Bulling, Andreas; Roggen, Daniel; Tröster, Gerhard (D)

Publication date:

2009

Permanent link:

https://doi.org/10.3929/ethz-a-005783740

Rights / license:

In Copyright - Non-Commercial Use Permitted

Originally published in:

Journal of Ambient Intelligence and Smart Environments 1(2), https://doi.org/10.3233/AIS-2009-0020 


\title{
Wearable EOG goggles: Seamless sensing and context-awareness in everyday environments
}

\author{
Andreas Bulling*, Daniel Roggen and Gerhard Tröster \\ Wearable Computing Laboratory, ETH Zurich, Gloriastrasse 35, 8092 Zurich, Switzerland
}

\begin{abstract}
In this article we introduce the analysis of eye motion as a new input modality for activity recognition, contextawareness and mobile HCI applications. We describe a novel embedded eye tracker that, in contrast to common systems using video cameras, relies on Electrooculography (EOG). This self-contained wearable device consists of goggles with dry electrodes integrated into the frame and a small pocket-worn component with a DSP for real-time EOG signal processing. It can store data locally for long-term recordings or stream processed EOG signals to a remote device over Bluetooth. We show how challenges associated with wearability, eye motion analysis and signal artefacts caused by physical activity can be addressed with a combination of a special mechanical design, optimised algorithms for eye movement detection and adaptive signal processing. In two case studies, we demonstrate that EOG is a suitable measurement technique for the recognition of reading activity and eyebased human-computer interaction. Eventually, wearable EOG goggles may pave the way for seamless eye movement analysis in everyday environments and new forms of context-awareness not possible today.
\end{abstract}

Keywords: Wearable Eye Tracking, Electrooculography (EOG), Context-Awareness, Activity Recognition, Human-Computer Interaction $(\mathrm{HCI})$

\section{Introduction}

Fifteen years ago, ubiquitous computing was introduced as a vision of technology fading into the background and always ready to interact and transparently respond to the users' needs. To realise this vision it is essential to be able to recognise and react according to the users' context $[1,28]$. This is primarily a pattern recognition problem in which context is inferred from specific sensor signal patterns. Sensing the users' context may thereby rely on ambient infrastructure so-called smart environments - or on wearable sensing and computing [30]. Wearable sensing allows for permanent context recognition and enables access to aspects that are difficult to measure with ambient sensors. By combining both, ubiquitous systems can be

${ }^{*}$ Corresponding author. E-mail address: bulling@ife.ee.ethz.ch; Tel.: +41 44632 2996; Fax: +41 446321210 developed that provide seamless sensing across different environments the user visits in his daily routine.

Human activity is one of the key aspects of user context. The recognition of physical activity both using ambient [27,32] and body worn [4] sensors has been extensively studied. However, context-awareness encompasses more than mere physical activity. The inclusion of social and affective aspects may help to paint a more accurate view of the user's context [37]. Cognitive aspects such as attention and intentionality may enhance activity recognition systems and benefit proactive wearable assistants. So far, most of these aspects remain unexplored as they cannot be picked-up by sensors usually deployed in today's wearable and ubiquitous computing scenarios.

A rich source of information about the state of the user can be found in the movement of the human eyes. This includes information related to the users' activities, such as reading [9], but also to cognitive processes of visual perception such as attention [26], saliency de- 
termination [19], visual memory [31] and perceptual learning [12]. Deliberate eye movements can be implemented for human-computer interaction (HCI) to provide feedback that can be used as explicit contextual information [8]. Attentive user interfaces (AUI) may also infer user intention and activity by analysing unconscious eye movements. As part of an ongoing project we investigate up to which extent deliberate and unconscious eye movements can be exploited to enable new kinds of context-aware applications.

The investigation of eye motion may focus on eye tracking or gaze tracking. Eye tracking refers to the analysis of general characteristics of relative eye movements, long-term movement dynamics or statistical properties of eye motion. In contrast, gaze tracking refers to the estimation of absolute gaze direction.

For stationary settings, a wide range of hardware for accurate gaze tracking is available. However, state-ofthe-art mobile systems still do not meet the requirements for unobtrusive long-term recordings and realtime analysis in everyday environments. To our knowledge, at this stage no wearable solution exists that is self-contained and unobtrusive for use in daily-life activities. Furthermore, current video-based systems are not geared to embedded online analysis of eye motion to recognise human activity and infer user context.

In this work, we address these issues by describing the design and implementation of a highly-integrated wearable eye tracker for context-awareness and mobile HCI based on Electrooculography (EOG). In contrast to well-established vision-based gaze tracking, EOG is measured with body-worn sensors and can be implemented as a low-cost and low-power embedded system. The device allows for unobtrusive recordings of EOG signals and their real-time processing and enables online inference of activity and context. It consists of glasses and a light-weight device worn on the body and is particularly designed for long-term use in daily life with simultaneous physical activity.

The specific contributions of this work are (1) the design and implementation of a wearable EOG-based eye tracker implemented as goggles, (2) the development of a software framework for continuous EOG signal analysis and the detection of eye movement events, (3) the evaluation and implementation of a set of algorithms for robust EOG signal processing within this framework and (4) the characterisation of wearable EOG and the goggles in two case studies.

\subsection{Paper organisation}

Section 2 provides information on the physiology of eye motion and the state-of-the-art in eye movement research with particular emphasis on sensors and applications. Based on the definition of the requirements for a wearable EOG-based eye tracking device, in Section 3, we describe the implementation challenges and the final design of the wearable EOG goggles. Section 4 gives detailed information on the signal processing algorithms implemented on the device for continuous recognition of eye movement sequences. Two case studies enabled by wearable EOG and the goggles are outlined in Section 5. In Sections 6 and 7, we discuss the current status of the system, comment on the case studies and give an outlook to future work, respectively.

\section{Eye movements: physiology, sensors and applications}

\subsection{Physiology of eye motion}

To be able to take advantage of the typical characteristics of eye movements to perform context recognition and implement eye-based HCI, it is important to understand its two main types, namely saccades and fixations.

Saccades: Humans do not look at a wider scene in a steady way. Instead, their eyes move around constantly to locate interesting parts and combine them into one mental representation. The main reason for this is that only a small central region of the retina, the fovea, allows to perceive the scene with high acuity. Simultaneous movements of both eyes in the same direction are called saccades. Typical characteristics of saccadic eye movements are $400^{\circ} / \mathrm{s}$ for the maximum velocity, $20^{\circ}$ for the amplitude and $80 \mathrm{~ms}$ for the duration [16].

Fixations: A fixation is the static state of the eyes during which gaze is held upon a specific location. Humans typically alternate saccadic eye movements and fixations (see Figure 1). However, visual fixation is never perfectly steady and fixational eye movements can also occur involuntarily. The term "fixation" can also be referred to as the time between two saccades during which the eyes are relatively stationary. Certain activities involve characteristic fixation patterns. For example during reading, the eyes fixate on successive locations within a line but also across a page to reach different sections of the text [9]. 


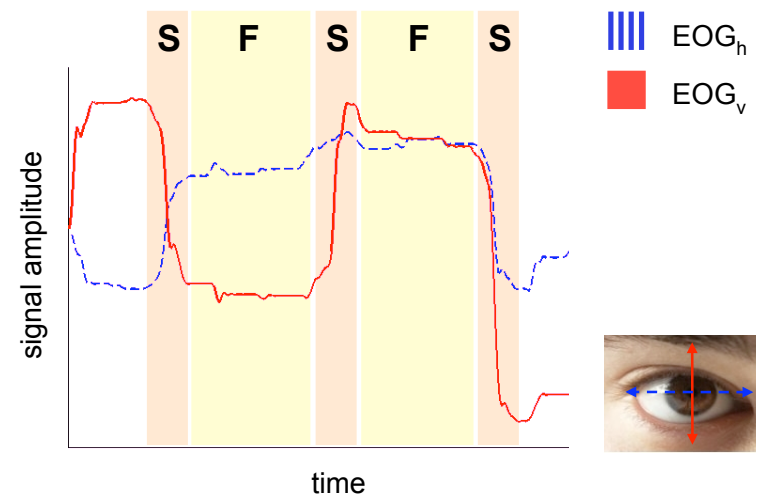

Fig. 1. Denoised horizontal $\left(E \mathrm{GG}_{\mathrm{h}}\right)$ and vertical $\left(\mathrm{EOG}_{\mathrm{v}}\right) \mathrm{EOG}$ signals with marked segments showing the two main eye movement characteristics: saccades (S) and fixations (F).

\subsection{Eye movements in daily life}

A growing number of researchers investigate movements of the eyes during daily activities. Important advances have been made to understand how the human brain processes visual tasks [18], how vision contributes to the organisation of active tasks in everyday life [25] and how eye, head, and hand movements are coordinated temporally [34]. In a recent study, Logan et al. studied activity recognition with a person living in a smart environment instrumented with a large number and variety of common sensors [27]. They found that among all activities, reading was one of the most difficult to detect. They concluded that in order to catch all types of physical activity in daily-life scenarios, novel sensors and algorithms need to be developed.

Although eye movements have been investigated in daily routine, none of these studies used eye movement patterns to perform activity and context recognition. Furthermore, other aspects of visual perception such as attention [26], saliency determination [19], visual memory [31] or perceptual learning [12] so far remain unexplored as a novel input for context-aware systems.

\subsection{Video-based eye tracking}

\subsubsection{Devices}

The common method to track eye gaze in natural environments are systems based on video cameras. A number of commercial gaze trackers are available of which some are targeted at mobile use, for example the Mobile Eye from Applied Science Laboratories (ASL) [2] or the iView X HED from SensoMotoric Instruments (SMI) [40]. Nevertheless, they still require bulky headgear and additional equipment to process the video streams. This does not allow for unobtrusive recordings and constrains the user in his physical activities. Furthermore, as the video processing is performed in real-time requiring considerable computational power, these systems are limited to only very few hours of mobile gaze tracking.

\subsubsection{Eye-based interaction}

Eye-gaze recorded using vision has long been investigated as a means to interact with a computer. However, due to the lack of appropriate hardware, mobile eye-based interaction is a so far barely regarded field of research. Most HCI work has focused on direct manipulation of user interfaces in stationary settings. Zhai et $a l$. proposed a new way of using eye gaze for computer input [46]. Results of an early-stage experiment indicated that their method potentially reduces physical effort and fatigue. Qvarfordt et al. explored an interactive human-computer dialogue system for touristic city trip planning [36]. They showed that it was possible to sense the users' interests based on eye-gaze patterns and adapt the system's output accordingly. Drewes et al. proposed to use eye gestures to implement new ways of HCI [15]. They argue that these gestures are insensitive to accuracy problems and do not exhibit the "Midas touch" problem (for details see [21]).

\subsection{EOG-based eye tracking}

\subsubsection{Electrooculography (EOG)}

The eyes are the origin of a steady electric potential field, which can also be detected in total darkness and if the eyes are closed. It is generated by a dipole with its positive pole at the cornea and its negative pole at the retina. The magnitude of this so-called corneo-retinal potential difference (CRP) lies in the range of $0.4 \mathrm{mV}$ to $1.0 \mathrm{mV}$. On the assumption of an unchanging CRP, the electric signal that can be derived using two pairs of skin electrodes placed at periorbital positions around one eye is called Electrooculogram (EOG). EOG typically shows signal amplitudes ranging from $5 \mu \mathrm{V} /{ }^{\circ}$ to $20 \mu \mathrm{V} /{ }^{\circ}$ and an essential frequency content between $0 \mathrm{~Hz}$ and $30 \mathrm{~Hz}$ [7]. If the eyes move from the centre position towards the periphery, the retina approaches one electrode while the cornea approaches the opposing one. This change in the orientation of the dipole and the electric potential field results in a change in the measured EOG signal. Inversely, by analysing these changes, eye movements can be tracked. 
Baseline drift: Baseline drift is a slow signal change mostly unrelated to the actual eye movements but superposing the EOG signal. Baseline drift has many possible sources as for instance interfering background signals, electrode polarisation [17] or physical influences such as varying contact pressure of the electrodes. The differences in EOG signal amplitude during saccadic eye movements can be assumed to be drift-free as saccades are performed in a very short period of time. All other signals can become subject to changes caused by baseline drift. In a four electrode setup, baseline drift can be different for the horizontal and vertical EOG signal components.

Several approaches to remove baseline drift from electrocardiographic signals (ECG) have been proposed in recent literature (for example see [41,11]). As ECG shows repetitive characteristics, some of the algorithms perform sufficiently well at removing baseline drift from these signals. However, they perform worse for signals with non-repetitive characteristics such as EOG. Thus, the development of robust algorithms for baseline drift removal from EOG signals is still an active field of research.

\subsubsection{Devices}

Efforts to miniaturise video-based eye trackers led researchers to consider EOG signals recorded with standard equipment for eye tracking in stationary settings $[20,6]$. Others investigated novel electrode configurations for implementing EOG-based eye trackers for mobile use. Manabe et al. proposed a system that uses EOG electrode arrays mounted on ordinary headphones [29]. While this approach might be less obtrusive than electrodes stuck to the face it raises other issues - namely, low signal-to-noise ratio (SNR) and poor separation of the horizontal from the vertical component of eye motion. Vehkaoja et al. presented a light-weight head cap for EOG and facial EMG measurements with electrodes embroidered of silver coated thread [42]. A small device integrated into the cap allows for wireless data transmission. Signal processing is performed offline using a standard desktop computer and the system is still to be evaluated in operation.

Although novel devices for recording eye motion using EOG have been developed, none of them allows for combined, embedded signal acquisition and activity and context recognition in a standalone wearable device. The system described in this work is a highly miniaturised, autonomous wearable sensor particularly designed for both tasks. Low-power and light-weight implementation, real-time signal processing capabilities and additional sensors for artefact compensation make this embedded system a unique solution for robust recordings in mobile daily life settings.

\subsubsection{EOG-based interfaces}

Basic eye movement characteristics detected from EOG signals such as saccades, fixations, blinks and deliberate movement patterns have been used for handsfree operation of stationary human-computer [13,22] and human-robot $[23,10]$ command interfaces. As part of a hospital alarm system, EOG-based switches provided immobile patients with a safe and reliable way of signalling an alarm [43]. All of these studies show that EOG is a measurement technique that is easy to operate, reliable and can also be made cosmetically acceptable.

For mobile settings, EOG-based interfaces have been developed for assistive robots [45] and as a control for an electric wheelchair [5,35]. These systems are intended to be used by physically disabled people who have extremely limited peripheral mobility but still retain eye motor coordination. Mizuno et al. used basic characteristics of eye motion to operate a wearable computer system for medical caregivers [33]. Although these studies target mobile settings the people themselves are still constrained in their movements.

\subsection{Summary}

In this section, we have shown that the eyes are a rich source of information which has not yet been used for activity and context recognition or mobile HCI applications. A review on the state-of-the-art in eye tracking devices and related applications revealed that the main reasons for this is the lack of an appropriate sensor and missing processing techniques for online context recognition based on eye motion.

We have shown that EOG provides several advantages over common systems based on video in particular in terms of embedded implementation and longterm recordings in daily life. However, in current work the information obtained from EOG remains coarse, the users are static, and signal processing is done offline using desktop computers. In this work we demonstrate how complex contexts can be recognised from EOG in mobile scenarios using an autonomous wearable device without the need for such additional infrastructure. 


\section{Design and implementation of a wearable context-aware EOG sensor}

\subsection{Requirements}

A wearable context-aware eye tracking device based on EOG that is robust to simultaneous physical activity and changing environments has to meet the following requirements:

1. To achieve a convenient and unobtrusive implementation and minimise user distraction the device needs to be wearable and light-weight.

2. To allow for autonomous long-term recordings the device needs to be low-power and support onboard data storage.

3. The device needs to provide real-time signal processing capabilities to allow for context-aware interaction.

4. To compensate for EOG signal artefacts caused by physical activity and changes in ambient light [7] an accelerometer and a light sensor need to be added.

Given the fact that EOG is recorded using electrodes placed around the eye, the natural choice for the basic form of the device was that of goggles: A goggles frame is built to minimise distraction to the user. It is very close to the face and covers the lateral positions on each side of the head commonly used for placing the electrodes. Only for the electrodes recording the vertical signal component above and below the eye an extension to the frame needs to be made. Furthermore, a goggles frame provides enough space to carry a small component that contains the amplification circuits for the analogue signals, the accelerometer and a connection to the light sensor. The latter can be fixed to the frame in between both eyes to provide measurements of incident light.

\subsection{Challenges}

The first trade-off we had to deal with was the one between wearability and signal quality: To reduce noise, the analogue amplification circuit and conversion from analogue to digital signals must occur close to the electrodes integrated into the frame. However, this results in increased weight and size and therefore reduced wearability. To optimise the design for weight, only the light sensor, the accelerometer and the amplification circuits can be attached to the glasses frame. On the downside, due to a longer wire up to the pro- cessing unit, in this case the analogue high-impedance EOG signals pick up an increased amount of noise compared to the first option.

A second challenge was the decision for the type of electrodes and their mounting to the glasses frame. Wet electrodes are commonly used for EOG recordings and provide high quality trace pickup. They come with a built-in layer of conductive gel, which assures good conductivity between the skin and the electrodes to minimise signal artefacts. However, because of the gel layer, they need to be stuck to the face with an adhesive brim, which may be uncomfortable and potentially irritate the skin.

In contrast to wet electrodes, dry electrodes are more convenient as they allow for easy removal and reattachment to the skin. However, they can move on the skin or can loose contact. Proper signal acquisition therefore requires a mechanical mounting that assures permanent contact and constant pressure on the skin at any time. To accommodate for different head sizes the electrode mounting also needs to be adjustable. Even if good electrode-skin contact can be guaranteed, dry electrodes usually show lower signal stability. This is caused by a higher initial contact resistance, which decreases considerably over time thus resulting in increased signal drift (see Section 2.4.1).

The described challenges emphasise that a proper design of an integrated wearable sensor based on EOG is subject to a variety of sometimes contradictory technical constraints. We believe, however, that most of these can be solved in the future and therefore decided to optimise the first prototype of the wearable eye tracker for wearability and comfort accepting a mechanically more complex device and potentially lower signal quality.

\subsection{Hardware design}

The final design consists of two components (see Figures 2, 3 and 4): Goggles with integrated electrodes and a signal processing unit (called WEPU, Wearable EOG Processing Unit).

The complete system weighs $188 \mathrm{~g}$ (Goggles: $60 \mathrm{~g}$, WEPU: $78 \mathrm{~g}$, cable: $50 \mathrm{~g}$ ) and is powered by a $3.7 \mathrm{~V} /$ $1500 \mathrm{mAh}$ Li-polymer battery attached to the WEPU. The total power consumption is $769 \mathrm{~mW}$ when storing data on the MMC card and $828.4 \mathrm{~mW}$ when streaming data to a remote computer using Bluetooth. This allows for up to 7.2 hours (MMC) and 6.7 hours (Bluetooth) of autonomous eye movement recording, respectively. 


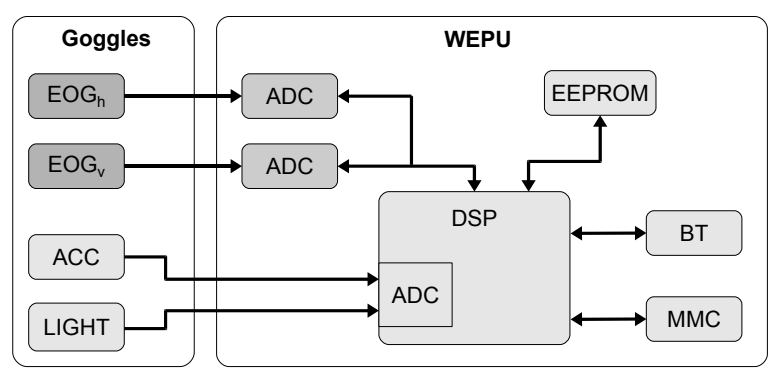

Fig. 2. Two-part hardware architecture of the EOG-based wearable eye tracker with EOG amplification circuitry $\left(\mathrm{EOG}_{\mathrm{h}}, \mathrm{EOG}_{\mathrm{v}}\right)$, accelerometer (ACC), light sensor (LIGHT), analog-digital converters (ADC), DSP, EEPROM, Bluetooth module (BT) and MMC card holder for data transmission and storage.

\subsubsection{Mechanics}

The Goggles contain the light sensor, dry EOG electrodes and a small analogue amplification board (see Figure 3). The light sensor is attached at the front of the frame in between both eyes pointing forward in line of incident light. Although a minimum of five EOG electrodes is required for recording eye motion (four for the two signal components and one for the reference signal), six electrodes are arranged around both eyes. This allows for flexibility if one of the eyes can not be measured due to poor signal quality. The electrodes are mounted on spring steel to ensure permanent skin contact and constant contact force. The spring steel is bent in such a way that the electrodes are placed flat on the skin. Because of the anatomy of the skull, this is particularly challenging for the electrodes above and below the eye, which need to be placed further away from the frame. Each electrode is connected to the amplification board with a shielded one core cable following the frame's shape. The amplification board has a size of $42 \times 15 \mathrm{~mm}$ and is screwed onto the left side of the frame. Besides the analogue amplification circuit this board also contains an accelerometer for measuring head movements. The Goggles are connected to the WEPU with a shielded 14 core cable. The WEPU can be worn on the body, e.g. in a cloth bag fixed to one of the upper arms.

\subsubsection{Electronics}

The EOG signal is composed of a small voltage superimposed by a large offset voltage relative to the ground electrode above the right eye. The offset is mostly caused by stray electrical signals on the leads and therefore referred to as common-mode interference. If an electric circuit is able to efficiently reject this interference it has a high common-mode rejection ratio (CMRR). For signal amplification on the Gog-

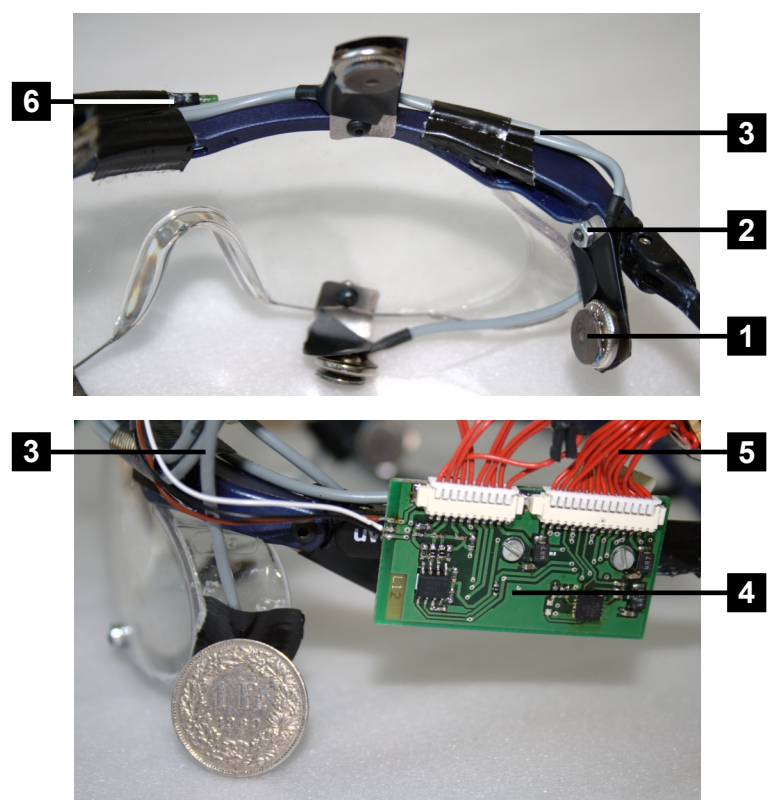

Fig. 3. Detailed view of the Goggles component: Dry electrodes mounted on spring steel (1) and screwed to the frame (2). Shielded one core cables (3) connect each electrode to a small analogue amplification board (4), which is connected with a shielded 14 core cable (5) to the WEPU. The pictures also show the light sensor attached at the front of the frame (6).

gles, we decided for low-power, medical grade instrumentation amplifiers. They provide a very high CMRR rating of $110 \mathrm{~dB}$ and $10^{10} \Omega$ input impedance and thus meet the requirements for a potentially robust signal acquisition. To further increase the CMRR, a Driven Right Leg (DRL) circuit [44] is implemented on the Goggles. This circuit measures the common mode potential and feeds its negative back into the body to actively cancel signal interference. Using this approach, we are able to achieve a CMRR of more than $105 d B$.

The WEPU is the core signal processing unit of the system with a credit card size of $82 \times 56 \mathrm{~mm}$ (see Figure 3). It is based on a 16-bit dsPIC from Microchip and contains dedicated 24-bit Delta-Sigma ADCs for each EOG channel, a Bluetooth and a MMC module and an EEPROM. The main advantage of the dsPIC compared to other microcontrollers commonly used on wearable sensors is its suitability for efficient real-time signal processing. It runs at $3 \mathrm{~V}$ with 40 Million Instructions Per Second (MIPS). The ADCs are critical for fast analog-digital conversion with high resolution. To achieve a resolution of $2.5 \mu \mathrm{V} /{ }^{\circ}$ for EOG signals with a dynamic range of $600 \mathrm{mV}$ the ADCs have to provide a minimum resolution of $18 \mathrm{bit}$. On the wearable eye tracker, the two ADCs allow the raw EOG 
signals to be processed with a sampling rate of up to $250 \mathrm{~Hz}$ and a resolution of 20 bits noise-free, i.e. that can be distinctly resolved. Processed data can either be transmitted using Bluetooth or stored on the MMC for offline analysis. The EEPROM is used to store configuration data and parameters for the signal processing algorithms described in Section 4. Four LEDs and two buttons allow the user to access the functionality of the device.

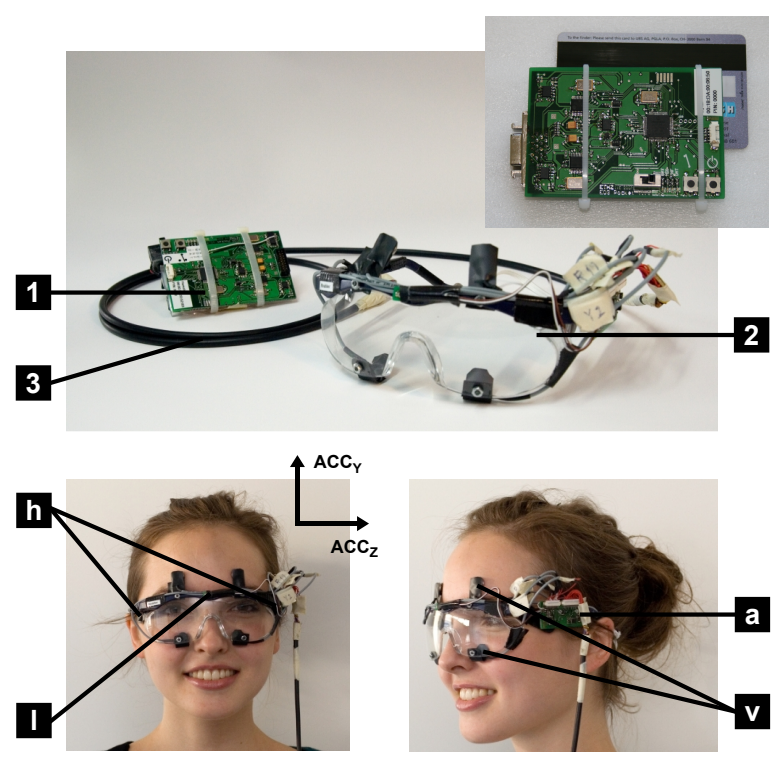

Fig. 4. Components of the EOG-based wearable eye tracker: the $W E P U$ with credit card size (1), the Goggles (2) and the shielded 14 core cable (3). The pictures at the bottom show the Goggles worn by a person with the positions of the two horizontal (h) and vertical (v) dry electrodes, the light sensor (1) and the accelerometer (a) with direction of its axes $\left(\mathrm{ACC}_{\mathrm{Y}}, \mathrm{ACC}_{\mathrm{Z}}\right)$

\subsection{Firmware}

The dsPIC on the WEPU runs freeRTOS, an opensource real-time operating system devised for embedded systems. freeRTOS is configured to run in preemptive mode using predefined task priorities. Using an operating system does not only contribute to clean and well-structured code but also provides services such as interrupt handling and a scheduler, which eases development and later code maintenance. In addition, this allows us to eventually run a context recognition middleware and opens up the possibility to integrate the device into multi-modal context recognition systems, physiological sensor networks or smart sensing environments. As a first step in this direction, we have im- plemented a driver for the Context Recognition Network (CRN) Toolbox [3], which enables the recording of EOG for eye movement analysis to be automatically synchronised with signals coming from body-worn accelerometers for activity recognition.

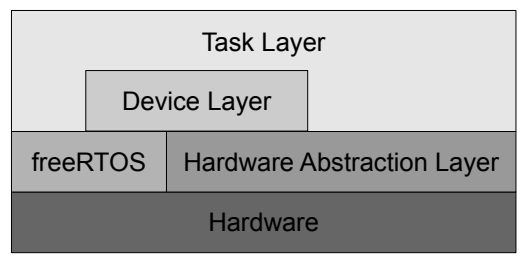

Library

Fig. 5. Three-tier software architecture of the EOG-based wearable eye tracker with layers for hardware abstraction and the operating system (freeRTOS), access to external components (Device Layer), common routines (Library) and core functionality (Task Layer).

The firmware is composed of three layers (see Figure 5). Among these layers, the Hardware Abstraction Layer (HAL) accesses the hardware. It provides a number of interfaces to the upper layers thus hiding all low-level hardware access. The Device Layer (DEL) uses the HAL to provide functionality for components external to the DSP such as the Bluetooth and the MMC module. For the MMC, a custom file system has been implemented to allow for efficient data storage. The binary EOG data can afterwards be converted into a standard two-column format using a custom software. The core functionality of the firmware is provided by the following five freeRTOS tasks implemented in the Task Layer (TAL):

The Controller Task is in charge of the interface components, power management and task control. It processes events coming from the two push buttons and the LEDs, monitors the output of the charger IC and activates the stand-by mode. In this mode, it reduces the DSP clock speed, holds the Bluetooth module and MMC in reset state and powers down the ADCs.

The Packet Reader Task reads packets received from the Bluetooth module, calculates a checksum and stores valid packets into a receive buffer. If the task receives a configuration packet, it initiates the reconfiguration process during which the Sampler Task and Processing Task may select other types or a different order of the algorithms for EOG signal processing.

The Sampler Task retrieves data samples from the external 24-bit ADCs and uses the 12-bit ADC internal to the DSP to sample the accelerometer and light sensor signals. The data is then passed on to the Processing Task. 
The Processing Task implements the core functionality of the eye tracker as it takes samples from the Sampler Task, executes each building block of the signal processing cascade and passes processed samples on to the Communicator Task. The type and order of the processing algorithms applied to the signals within the cascade can freely be chosen by the user (see Figure 6 for the default).

The Communicator Task reads data from the sample queue, builds a data packet and sends the packet either to the MMC controller or the Bluetooth module depending on the output medium selected by the user. It is also in charge of operating these peripherals.

Additionally, a separate Library contains functionality that is shared by these tasks such as the CRC routines.

\section{EOG signal processing for context sensing}

In this section, we describe the signal processing cascade implemented on the wearable eye tracker (see Figure 6). The processing is tailored to the particular needs of recognising context information from EOG signals. It aims at removing artefacts and noise from the EOG signals. Additionally, it aims at providing robust detection of saccades and blinks for eye movement event encoding and the recognition of socalled eye gestures, which consist of several consecutive movements. We first describe the denoising filter and the algorithm for compensation of EOG signal artefacts caused by walking. We continue with a description of the algorithms for the detection of blinks and saccades, removal of blinks, eye movement event encoding and eye gesture recognition.

\subsection{Adaptive Filtering}

\subsubsection{Denoising}

Raw EOG signals are corrupted with noise from the following sources:

- Noise caused by the residental powerline, usually referred to as mains hum

- Noise introduced by the measurement circuitry, the electrodes, wires, etc.

- Noise from other physiological sources interfering with EOG such as electromyographic (EMG) signals

- Noise due to simultaneous physical activity, which may cause the electrodes to loose contact or move on the skin

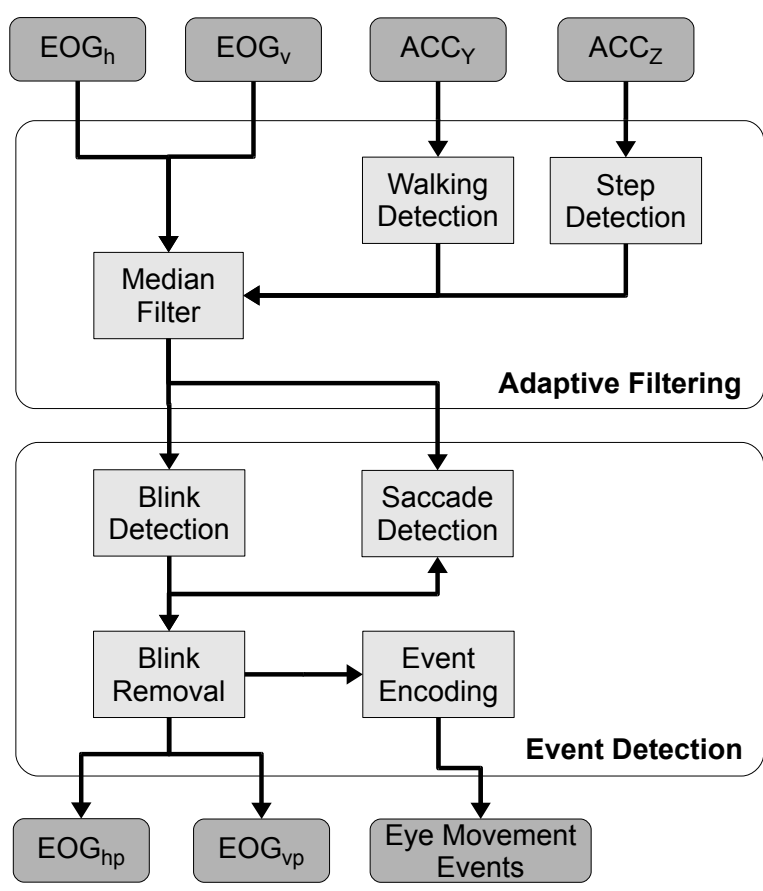

Fig. 6. Flowchart showing the building blocks of the signal processing cascade implemented on the wearable eye tracker: horizontal and vertical EOG $\left(\mathrm{EOG}_{\mathrm{h}}, \mathrm{EOG}_{\mathrm{v}}\right)$ and acceleration $\left(\mathrm{ACC}_{\mathrm{Y}}, \mathrm{ACC}_{\mathrm{Z}}\right.$, cf. Figure 4) signals as input, adaptive filtering using a median filter and eye movement event detection. As output, the system returns the detected eye movement events as well as the processed EOG data $\left(\mathrm{EOG}_{\mathrm{hp}}, \mathrm{EOG}_{\mathrm{vp}}\right)$.

As a first step in the signal processing cascade, noise reduction is necessary to improve the signal for the following processing blocks. In contrast to other physiological signals such as those from electrocardiography (ECG), eye movements are usually non-repetitive, which make the generated EOG signals unpredictable. This mostly prohibits to apply optimised algorithms that make use of structural and temporal knowledge about the expected signal to improve denoising quality. Furthermore, EOG signals exhibit characteristics that need to be preserved for further signal analysis. The signal edges need to be retained to allow for saccade detection and analyse eye movement dynamics. Signal amplitudes need to be preserved to allow to distinguish between different types and directions of saccadic eye movements. Denoising filters must not introduce artificial signals that may accidently be interpreted as eye movements in subsequent signal processing steps.

To identify a suitable approach for denoising we evaluated different algorithms, such as a standard lowpass filter, a filter based on wavelet shrinkage denoising (see [14] for details) and a standard median filter 
applied on a signal window with fixed length. From our experiments we found that the median filter performed best as it preserved edge steepness of saccadic eye movements, retained signal amplitudes and did not introduce any artificial signals. Furthermore, as the median filter is computationally light-weight it is well suited for online signal processing on a DSP. However, it is crucial to choose an appropriate window size to reduce noise without removing important EOG signal parts.

\subsubsection{Motion artefact compensation}

As EOG is measured with body-worn sensors, motion causes artefacts in the signals and affects eye movement detection. Walking is a common activity in everyday life. Thus, walking serves as a good test bench for investigating artefacts induced by body motion. Analyses showed that artefacts in the EOG signals occur periodically according to the step frequency. A median filter with fixed window size fails to eliminate these artefacts for different persons and walking speeds. A parameter sweep on the window size using example data recorded from several subjects revealed that the optimal size is strongly related to the temporal step length. Therefore, we use an algorithm implementing an adaptive filter. The idea is to exploit the repetitive characteristic of walking and adapt the window size of the median filter to the step length as long as walking activity is detected (see Figure 7).

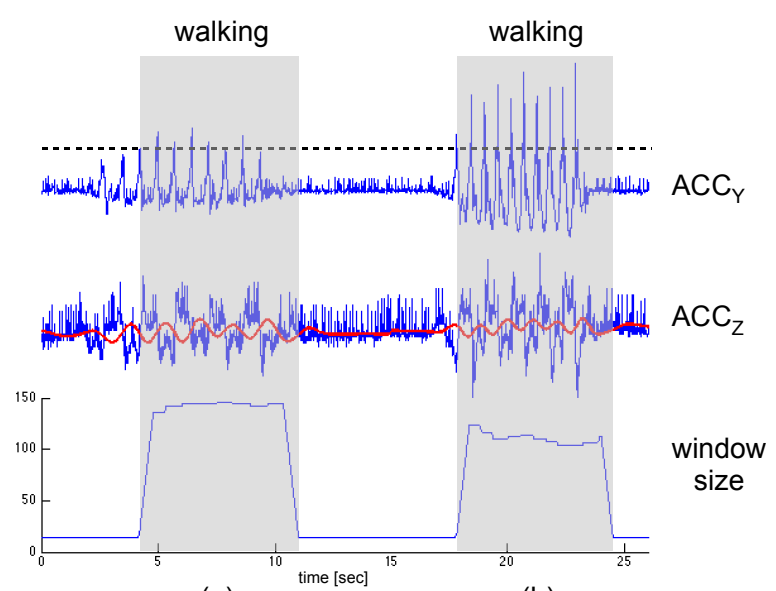

(a)

(b)

Fig. 7. Adaptive filter for artefact compensation while walking slowly (a) and fast (b). Vertical acceleration signal ( $\mathrm{ACC}_{\mathrm{Y}}$ ) and threshold (dashed line) for detecting walking activity. Horizontal acceleration signal $\left(\mathrm{ACC}_{\mathrm{Z}}\right)$ and first derivative for calculating the step length. The window size used by the median filter and tuned to the walking pace is shown at the bottom.
An approach for detecting footsteps using data from a head-mounted accelerometer has been described in [39]. To detect walking activity, we implemented an extended version of the algorithm. Our algorithm first analyses the vertical axis of the goggle-mounted accelerometer $\left(\mathrm{ACC}_{\mathrm{Y}}\right.$, cf. Figure 4$)$. If the corresponding signal exceeds a defined threshold, the algorithm tries to detect steps by searching for zero-crossings of the first derivative of the low-pass-filtered acceleration data of the horizontal axis ( $\mathrm{ACC}_{\mathrm{Z}}$, cf. Figure 4). Walking is assumed as long as such steps are detected. In order to smooth out variations in walking style for different subjects, the step length is calculated on the basis of three consecutive step movements (e.g. right left - right) separately for the left and the right leg. By calculating the length continuously for each step, the algorithm can adapt to different persons and walking speeds. For softer adaptation, only small increments are applied (see Figure 7). If walking activity is not detected anymore, the window size is progressively set towards its default value.

In [8] we evaluated the adaptive filter in a mobile setting. The experimental scenario involved subjects to perform different eye movements while standing and walking down a corridor. The expected eye movements were shown on a head-up display (HUD) with a defined order and timing. We recorded five male subjects between the age of 21 and 27 totalling roughly 35 minutes of recording with walking activity accounting for about 22 minutes. As the mobile setting did not allow to record a ground truth, we decided to do a comparison to a standard median filter with fixed window size to assess a relative performance measure. Figure 8 shows a boxplot for the total number of detected saccades in the horizontal EOG signal component. Each box summarises the statistical properties of the data of the subjects: The horizontal red lines in each box indicates the median and the upper and lower quartiles. The vertical dashed lines indicate the data range, points outside their ends are outliers. Boxes are plotted for the following cases: stationary and raw signal, stationary and fixed median filter, stationary and adaptive filter, walking and raw signal, walking and fixed median filter, walking and adaptive filter. The single solid horizontal line indicates the expected number of saccades defined by the experimental procedure. We found that the adaptive filter was able to reduce signal artefacts caused by walking activity by up to $80 \%$ in the horizontal, and up to $60 \%$ in the vertical signal component. 


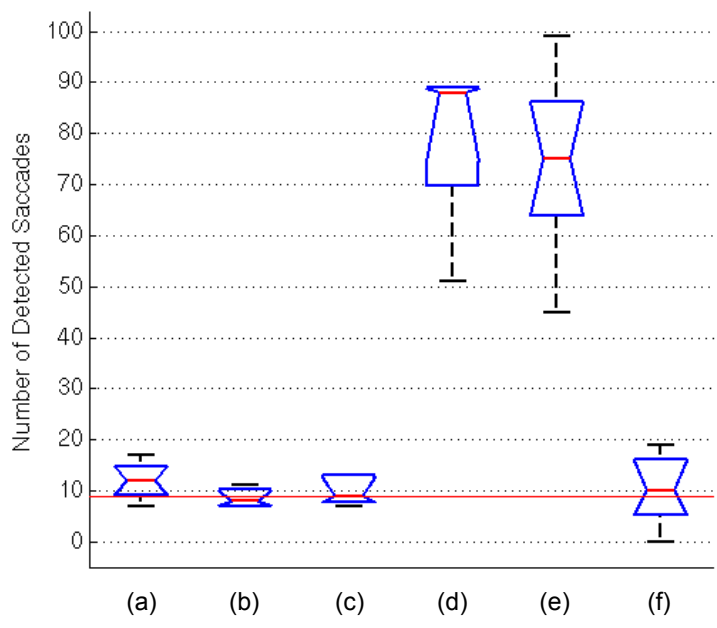

Fig. 8. Boxplot for the total number of detected saccades in the horizontal EOG signal component with fixed thresholds over all subjects: stationary/raw (a), stationary/fixed median filter (b), stationary/adaptive filter (c), walking/raw (d), walking/fixed median filter (e), walking/adaptive filter (f).

\subsection{Blink detection}

Blinks need to be detected in the vertical EOG signal component for two reasons: For certain HCI applications they may provide important input and a more versatile input alphabet. For applications focused on eye movement detection, blinks need to be detected and removed because their characteristics are very similar to those of saccadic eye movements. This can affect subsequent signal processing steps and eventually render robust eye movement analysis impossible.

We evaluated different algorithms with special attention to a real-time implementation on the DSP. The methods considered included continuous wavelet transform (CWT-BD), velocity threshold analysis [23], Haar wavelet decomposition [24] and template matching. Similar to the algorithm described in the following section, CWT-BD uses thresholding of wavelet coefficients for blink detection. The approach based on template matching works as follows: First, a blink template is created using manually cut equally-sized raw signal segments of 10 blinks from different persons, vertically shifted by their median and aligned at their peaks. To create the template, the mean at each sample point over all segments is calculated. Afterwards, blinks are detected by shifting this template over the vertical EOG signal component by following a sliding window approach. In each step, the Euclidean distance between the template and the signal segment of the current window is computed as a similarity metric. If the distance is below a defined threshold, i.e. the similarity between the template and the current segment is high, a blink event is recorded.

We evaluated each of these algorithms on nine EOG signals from different subjects (see Table 1). The signals contained a total of 105 blinks with different blink amplitudes (80 large, 25 small). For each algorithm, true positive $(T P)$, false positive $(F P)$ and false negative $(F N)$ counts were taken to calculate precision $\left(\frac{T P}{T P+F P}\right)$ and true positive rate (recall) $\left(\frac{T P}{T P+F N}\right)$ values. From the results we found that both CWT$\mathrm{BD}$ and template matching were able to recognise all large blinks while the velocity algorithm and the Haar wavelet performed worse. Although CWT-BD performed slightly better on blinks with smaller amplitude, for the wearable eye tracker we decided to use the template matching algorithm due to its higher precision rate. The reason is that a small number of blinks that are not recognised correctly have less negative impact on eye movement detection than the same number of actual movement events that get detected as blinks and removed due to wrong classification.

Table 1

Combined true positive $(T P)$, false positive $(F P)$, false negative $(F N)$, precision and true positive rate (recall) values for blink detection using different algorithms. The results were calculated based on nine EOG datasets from different subjects containing a total of 105 blinks of which 80 had large and 25 small amplitudes.

\begin{tabular}{lccccc}
\hline Algorithm & TP & FP & FN & Precision & Recall \\
\hline CWT-BD & 104 & 6 & 1 & $95 \%$ & $99 \%$ \\
Template Matching & 101 & 1 & 4 & $99 \%$ & $96 \%$ \\
Velocity Threshold & 91 & 0 & 14 & $100 \%$ & $87 \%$ \\
Haar Wavelet & 76 & 6 & 29 & $93 \%$ & $72 \%$ \\
\hline
\end{tabular}

\subsection{Saccade detection}

For saccade detection we developed the so-called Continuous Wavelet Transform - Saccade Detection (CWT-SD) algorithm, which can be efficiently implemented for real-time processing on the DSP. The CWT-SD first computes the continuous 1-D wavelet coefficients from the signal at scale 20 using Haar wavelets. Saccades are detected for all samples where the absolute value of the coefficient vector exceeds a defined threshold. The direction and size of a saccade is given by the sign of the first derivative and the maximum value of the corresponding EOG signal amplitude. 


\subsection{Blink removal}

For blink removal, the streams of saccade and blink events are analysed in parallel. Three cases need to be distinguished to maintain essential signal characteristics such as saccade amplitude and slope required for eye movement detection.

Presaccadic blinks are caused by blinks that share their last edge with a saccade. Presaccadic blinks are removed by replacing the blink interval with the signal value at the beginning of the blink.

Intersaccadic blinks usually occur during slow eye movements or fixation periods. This type of blink is removed by replacing its interval with a linear interpolation between the value at the beginning and the value at its end.

Postsaccadic blinks are blinks that immediately follow a saccade and thus share their first edge with it. For removal, the blink interval is replaced with the signal value at the end of the blink.

\subsection{Eye gesture recognition}

The idea of combining a sequence of distinct relative eye movements to create more complex gestures was introduced in [15] for a video-based eye tracker. We follow a similar approach for the continuous recognition of eye gestures based on EOG (see Figure 9). Our algorithm takes the streams of saccade events for the horizontal and the vertical EOG signal component as its input. It maps these saccades to eye movements with basic, intermediate and diagonal directions and finally encodes them into a combined string sequence. Basic directions are left, right, up and down (L, R, U, D). Diagonal eye movements $(1,3,7,9)$ are characterised by simultaneous saccades with similar signal amplitudes. If the saccades have different signal amplitudes, the corresponding eye movements are called intermediate (e.g. V, M).

The algorithm for eye movement detection works as follows: It first checks for simultaneous saccade events in both EOG signal components within a time window of $0.06 \mathrm{~s}$ given by eye physiology. If no simultaneous saccade events are detected, the single saccade is directly mapped to the symbol of the corresponding basic eye movement. If two such events are detected within the time window, a non-basic eye movement has occurred. The algorithm then uses the corresponding saccades' directions and amplitudes to combine both events into the appropriate symbol (c.f. Figure 9): Two saccades with equally large amplitudes are merged to the symbol exactly in between (e.g. symbols R and D are mapped to symbol 3). If the saccades' amplitudes differ by more than $50 \%$ the saccades are merged to the closest neighbouring symbol (e.g. symbols $\mathrm{r}$ and $\mathrm{D}$ are mapped to symbol V). This scheme encodes each eye movement into a distinct event symbol, thus merges both EOG signal components into one string sequence.
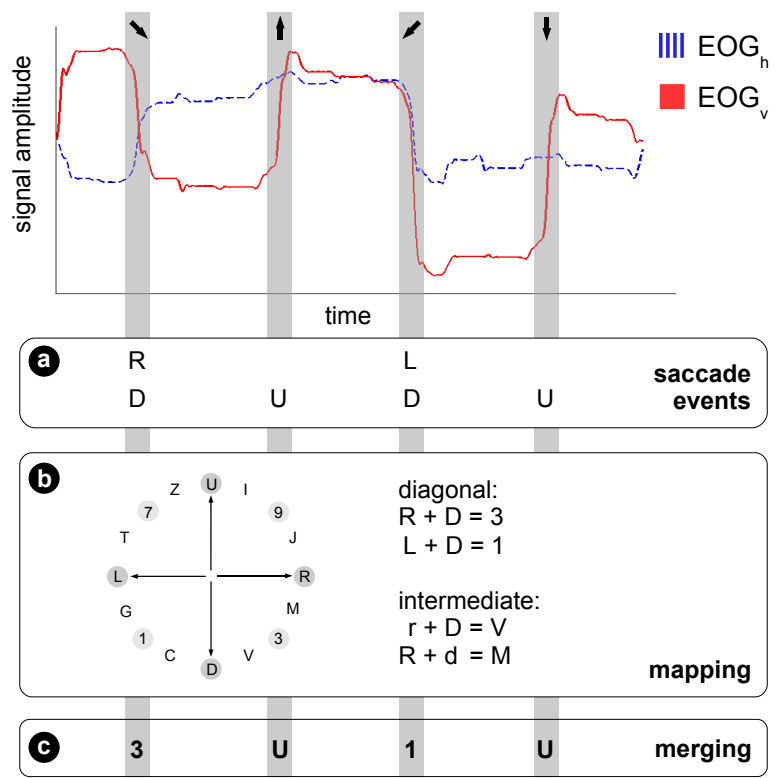

Fig. 9. Eye movement encoding from horizontal $\left(\mathrm{EOG}_{\mathrm{h}}\right)$ and vertical $\left(E G_{v}\right)$ EOG signals for gesture 3U1U: Windows marked in grey with distinct saccade events $(\mathrm{R}, \mathrm{L}, \mathrm{D}, \mathrm{U})$ detected in the horizontal and vertical signal component (a), mapping to basic (U) and diagonal $(1,3)$ eye movements (b) and final merging into a combined sequence of symbols (c). The circle in the middle shows all possible symbols for saccade event mapping.

To recognise eye gestures consisting of several consecutive movements, the resulting string sequence is scanned for eye movement patterns following a string matching approach: For matching, the sequence is continuously compared with templates representing all gestures required by the specific application. For each template, the edit distance between the templates and the segment is calculated. To allow for variability inherent in the eye gestures, the edit distance between two symbols of which one represents an intermediate direction is set to zero if the angle between them is smaller than or equal to $22.5^{\circ}$. If one of the templates exactly matches the current segment of the string sequence (i.e. the edit distance is zero), the corresponding eye gesture is recognised. 


\section{Case Studies}

\subsection{Activity recognition}

The aim of the experiment conducted in [9] was to recognise the reading activity of people in transit in an everyday environment using a wearable EOG system. We defined a scenario of travelling to and from work containing a semi-naturalistic set of reading activities. It involved eight subjects reading text while being engaged in a sequence of activities such as sitting at a desk, walking along a corridor, walking along a street, waiting at a tram stop and riding a tram. For this twoclass classification problem, we evaluated three recognition algorithms - string matching and two variants of Hidden Markov Models (HMMs), mixed Gaussian and discrete - on a dataset of about six hours.

Two leave-one-out training schemes were used for the HMMs: subject-dependent only using the calibration data from the subject being tested and subjectindependent using calibration data only from other subjects. The different methods were compared across a sweep of their main parameters. The resulting $\mathrm{Re}$ ceiver Operating Characteristics (ROC) curves for the subject-dependent and the subject-independent case are shown in Figure 10. These plot true positive rate against false positive rate (FPR) $\left(\frac{F P}{F P+T N}\right)$. Best case results approach the top left corner while worst case (which means random) follow the diagonal.

The ROC shows that string matching outperforms the HMMs. At its "best", we were able to recognise reading activity over all subjects using string matching with a recall of $71.0 \%$ and FPR of $11.6 \%$ (total accuracy $80.2 \%$ ). The mixed Gaussian returns a lower bestcase at recall $62.0 \%$, FPR $24.0 \%$ and accuracy $68.9 \%$ while the worst performing algorithm is the discrete HMM. The experiment has shown that wearable EOG is a feasible approach for recognising reading in dailylife scenarios and is robust across an example set of activities for different subjects.

\subsection{Human-computer interaction}

In [8] we evaluated the wearable eye tracker for human-computer interaction. To investigate the use of explicit eye gestures as a control input, we developed a desktop computer game consisting of eight different game levels. In each game level, subjects had to repeatedly perform a defined eye gesture as fast as possible until the first successful try (see Table 2). To reach a high score, wrong eye movements, i.e. movements

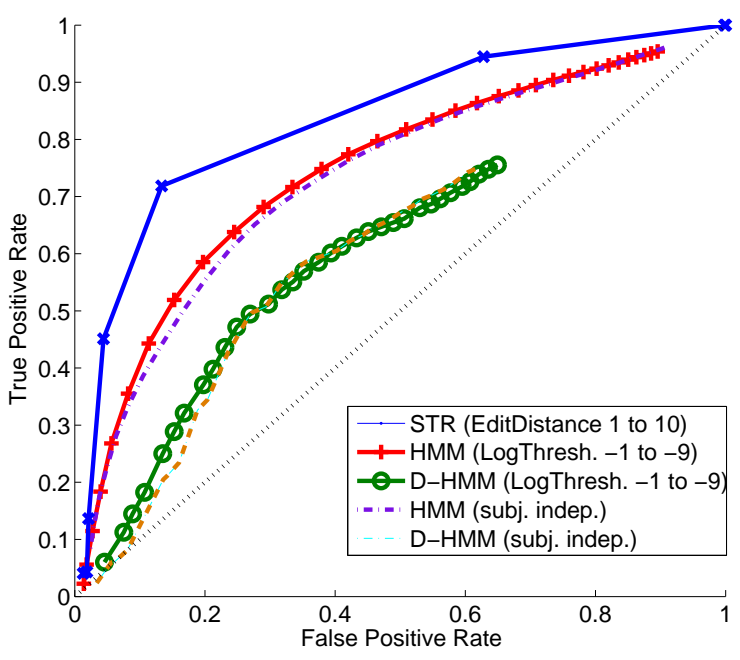

Fig. 10. ROC curves showing a performance comparison for the recognition of reading activity between string matching (STR), Gaussian HMM (HMM) and discrete HMM (D-HMM). For the HMMs, both the subject-dependent and the subject-independent results are shown.

that were not part of the expected gesture, had to be minimised. In contrast to a previous study [15], movements of the head and the upper body were allowed at any time. We collected data from eleven subjects - two female and nine male - between the ages of 24 and 64 .

Table 2

Eye gestures of increasing complexity and their string representations used for interaction in a computer game (cf. Figure 9). The grey dot denotes the start and the arrows the order and direction of each eye movement.

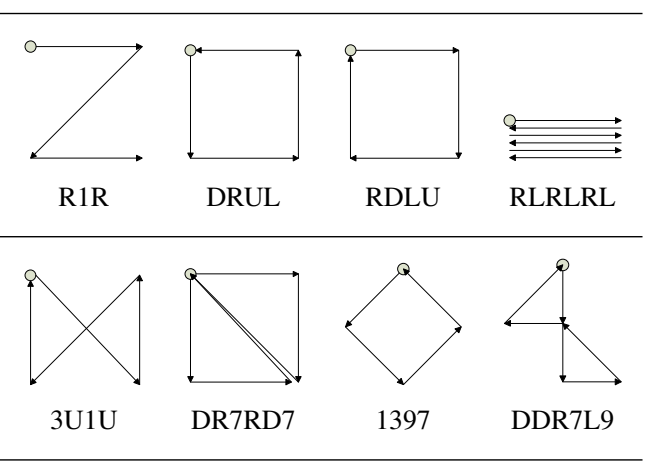

The average performance over all subjects is given in Table 3 which shows the times $T_{T}$ and $T_{S}$, the time ratio $T_{S} / T_{T}$ and the accuracy $A c c$ to perform each of the eight gestures. $T_{T}$ denotes the total time the subjects spent trying to complete each of the ges- 
tures while the success time $T_{S}$ only measures the time spent on all successful attempts. What can be seen from the table is that the subjects were able to achieve an average eye gesture accuracy of $87 \%$. Although the total time $T_{T}$ increased significantly for more complex gestures such as DDR7L9, the success time $T_{S}$ was comparable for all gestures. This highlights the fact that the eyes can be used as a versatile and fast input modality across gestures with different complexity.

Table 3

Average performance for the different gestures over all subjects. $T_{T}$ is the total time spent to complete the gesture and $T_{S}$ the success time spent only on successful attempts. The accuracy $A c c$ is the ratio of eye movements resulting in a correct gesture to the total number of movements performed until success.

\begin{tabular}{lcccc}
\hline Gesture & $T_{T}[m s]$ & $T_{S}[m s]$ & $T_{S} / T_{T}$ & $A c c[\%]$ \\
\hline R1R & 3370 & 2890 & 0.858 & 85 \\
DRUL & 4130 & 3490 & 0.845 & 90 \\
RDLU & 3740 & 3600 & 0.963 & 93 \\
RLRLRL & 6680 & 5390 & 0.807 & 90 \\
3U1U & 4300 & 3880 & 0.902 & 89 \\
DR7RD7 & 12960 & 5650 & 0.436 & 83 \\
1379 & 6360 & 3720 & 0.585 & 84 \\
DDR7L9 & 25400 & 5820 & 0.229 & 83 \\
\hline Average & 8368 & 4305 & 0.712 & 87 \\
\hline
\end{tabular}

\section{Discussion}

\subsection{On wearable eye tracking using EOG}

From our studies we found that the wearable eye tracker described in this work is a robust sensor for recording eye motion. In contrast to common systems using video, the device uses an EOG-based measurement technique that enables the unobtrusive implementation as goggles. The main advantage of EOG is the fact that the person only has to wear light-weight equipment. Our studies show that this contributes to the person feeling unconstrained and allows for natural behaviour and unrestricted physical activity in daily life settings. We plan to carry out additional experiments on human factors to further evaluate the goggles with respect to unobtrusiveness and user acceptance particularly for special user groups such as disabled or elderly people. Another advantage is that EOG processing requires less computational power than video due to lower data rates. This enables an embedded and low-power design and results in low data storage requirements. Both are crucial prerequisites for longterm data collection and real-time signal processing in mobile daily-life settings.

Our system particularly addresses challenges related to wearability, signal artefacts caused by physical activity and eye motion analysis. This is possible with a combination of a special mechanical design, adaptive signal processing and optimised algorithms for eye movement detection. An interesting question for future work is how the adaptive filter performs for people with different walking styles (e.g. elderly, persons limping, etc.) and how it can be extended to address different types of movement artefacts. A remaining issue with the current prototype is that dry EOG electrodes require permanent skin contact. Poor placement of electrodes and signal artefacts due to electrode movements were the reasons for many of the problems in our work. We believe that these may eventually be solved by developing special-purpose EOG goggles with a mechanically improved mounting that is tailored to assure permanent skin contact and proper electrode placement.

Baseline drift is an issue for wearable EOG recordings in particular if dry electrodes are used. It is for this reason that accurate eye-gaze tracking is difficult to achieve. Nevertheless, as we demonstrated in this paper, eye motion is a rich source of information on user activity and context that complements common pinpoint tracking. The development of novel electrodes is still a very active topic of research (for example see [38]). Eventually, dry electrodes that allow to record drift-free signals would allow EOG to be implemented for eye-gaze tracking.

\subsection{On the case studies}

The first case study shows that wearable EOG is a feasible approach for recognising reading activity in daily-life scenarios and is robust across an example set of simultaneous physical activities. We were able to detect reading activities over all subjects with a top recognition rate of $80.2 \%$. This result raises the question of whether different reading behaviours can be detected automatically. A "reading detector" could enable novel attentive user interfaces that take into account aspects such as user interruptability and level of task engagement.

Results from the second case study show that EOG is a robust modality for HCI applications that can be efficiently processed to recognise eye gestures consisting of several consecutive eye movements. While us- 
ing the eyes as a control input was quickly learned, $30 \%$ of the subjects reported of having had problems to stay concentrated during the game. However, fatigue is an intrinsic problem not only for eye gestures but also for common input modalities such as speech or hand gestures. Eye gestures outperform these modalities if the hands can not be used (e.g. during driving or while working on the computer) or if speech input is not possible (e.g. for privacy reasons or in very silent or very noisy surroundings).

\section{Conclusion and outlook}

In this work, we have demonstrated an autonomous EOG-based eye tracker and context recognition system integrated into goggles. We have shown that this unobtrusive device is applicable to different people and works in a wide range of applications. Its embedded and self-contained design allows for wearable sensing and online analysis of eye motion and extends these to everyday environments. This enables context-aware feedback, which is a key aspect to smart wearable assistants and smart environments. Furthermore, we have shown that the main characteristics of eye motion can be captured in an efficient way, which promises a range of new context-aware applications. EOG-based eye input allows for versatile human-computer interaction and may eventually provide new means of light-weight interaction for mobile settings.

The movement patterns our eyes follow in daily routine reveal much about what we are doing as well as what we intend to do. Our long-term objective is to investigate how much information eye motion can provide about the user's activity and context. By connecting several eye trackers, concurrent eye movement recordings for a group of people and distributed activity recognition may become possible. In addition, we also plan to investigate unconscious eye movements, which are the result of cognitive processes in the human brain. These processes are related to external aspects such as the user's activity or environment, but also to internal aspects of visual perception such as memory [31] and learning [12]. The analysis of eye motion thus may allow to deduce these aspects, which would give important input for future context-aware systems.

Eventually, eye motion may be used as a new sensing modality for activity recognition, contextawareness and mobile HCI applications, providing access to underlying cognitive processes not accessible with current sensing modalities.

\section{References}

[1] G. Abowd, A. Dey, P. Brown, N. Davies, M. Smith, and P. Steggles. Towards a Better Understanding of Context and Context-Awareness. In Proc. of the 1st International Symposium on Handheld and Ubiquitous Computing (HUC 1999), volume 1707, pages 304-307. Springer, 1999.

[2] Applied Science Laboratories (ASL). Mobile Eye. http: / /www. a-s-1.com/, 2009.

[3] D. Bannach, P. Lukowicz, and O. Amft. Rapid Prototyping of Activity Recognition Applications. IEEE Pervasive Computing, 7(2):22-31, April/June 2008.

[4] L. Bao and S. S. Intille. Activity Recognition from UserAnnotated Acceleration Data. In Proc. of the 2nd International Conference on Pervasive Computing (Pervasive 2004), pages 1-17. Springer, 2004.

[5] R. Barea, L. Boquete, M. Mazo, and E. Lopez. System for assisted mobility using eye movements based on electrooculography. IEEE Transactions on Neural Systems and Rehabilitation Engineering, 10(4):209-218, December 2002.

[6] D. Borghetti, A. Bruni, M. Fabbrini, L. Murri, and F. Sartucci. A low-cost interface for control of computer functions by means of eye movements. Computers in Biology and Medicine, 37(12):1765-1770, 2007.

[7] M. Brown, M. Marmor, and Vaegan. ISCEV Standard for Clinical Electro-oculography (EOG). Documenta Ophthalmologica, 113(3):205-212, nov 006.

[8] A. Bulling, D. Roggen, and G. Tröster. It's in Your Eyes - Towards Context-Awareness and Mobile HCI Using Wearable EOG Goggles. In Proc. of the 10th International Conference on Ubiquitous Computing (UbiComp 2008), pages 84-93. ACM Press, September 2008.

[9] A. Bulling, J. A. Ward, H. Gellersen, and G. Tröster. Robust Recognition of Reading Activity in Transit Using Wearable Electrooculography. In Proc. of the 6th International Conference on Pervasive Computing (Pervasive 2008), pages 19-37. Springer, May 2008.

[10] Y. Chen and W. S. Newman. A human-robot interface based on electrooculography. In Proc. of the International Conference on Robotics and Automation (ICRA 2004), volume 1, pages 243-248, April 26-May 1, 2004.

[11] V. S. Chouhan and S. S. Mehta. Total Removal of Baseline Drift from ECG Signal. In Proc. of the 17th International Conference on Computing Theory and Applications (ICCTA 2007), pages 512-515, March 5-7, 2007.

[12] M. M. Chun. Contextual cueing of visual attention. Trends in Cognitive Sciences, 4(5):170 - 178, 2000.

[13] Q. Ding, K. Tong, and G. Li. Development of an EOG (ElectroOculography) Based Human-Computer Interface. In Proc. of the 27th Annual International Conference of the Engineering in Medicine and Biology Society (EMBS 2005), pages 68296831, 2005.

[14] D. L. Donoho. De-noising by soft-thresholding. IEEE Transactions on Information Theory, 41(3):613-627, May 1995.

[15] H. Drewes and A. Schmidt. Interacting with the Computer Using Gaze Gestures. In Proc. of the 11th International Conference on Human-Computer Interaction (INTERACT 2007), pages 475-488. Springer, 2007.

[16] Andrew T. Duchowski. Eye Tracking Methodology: Theory and Practice. Springer-Verlag New York, Inc., Secaucus, NJ, USA, 2007. 
[17] J. J. Gu, M. Meng, A. Cook, and G. Faulkner. A study of natural eye movement detection and ocular implant movement control using processed EOG signals. In Proc. of the IEEE International Conference on Robotics and Automation (ICRA 2001), volume 2, pages 1555-1560, 2001.

[18] M. M. Hayhoe and D. H. Ballard. Eye movements in natural behavior. Trends in Cognitive Sciences, 9:188-194, 2005.

[19] J. M. Henderson. Human gaze control during real-world scene perception. Trends in Cognitive Sciences, 7:498-504, 2003.

[20] J. Hori, K. Sakano, M. Miyakawa, and Y. Saitoh. Eye Movement Communication Control System Based on EOG and Voluntary Eye Blink. In Proc. of the 9th International Conference on Computers Helping People with Special Needs (ICCHP), volume 4061, pages 950-953, 2006.

[21] R. J. K. Jacob. What you look at is what you get: eye movement-based interaction techniques. In Proc. of the SIGCHI Conference on Human Factors in Computing Systems (CHI 1990), pages 11-18. ACM Press, 1990.

[22] A. R. Kherlopian, J. P. Gerrein, M. Yue, K. E. Kim, J. W. Kim, M. Sukumaran, and P. Sajda. Electrooculogram based system for computer control using a multiple feature classification model. In Proc. of the 28th Annual International Conference of the Engineering in Medicine and Biology Society (EMBS 2006), pages 1295-1298, August 30-September 2006, 2006.

[23] Y. Kim, N. Doh, Y. Youm, and W. K. Chung. Development of human-mobile communication system using electrooculogram signals. In Proc. of the 2001 IEEE/RSJ International Conference on Intelligent Robots and Systems (IROS 2001), volume 4, pages 2160-2165, 2001.

[24] V. Krishnaveni, S. Jayaraman, S. Aravind, V. Hariharasudhan, and K. Ramadoss. Automatic Identification and Removal of Ocular Artefacts from EEG using Wavelet Transform. Measurement Science Review, 6(4):45-57, 2006.

[25] M. F. Land. Eye movements and the control of actions in everyday life. Progress in Retinal and Eye Research, 25(3):296324, 2006.

[26] S. P. Liversedge and J. M. Findlay. Saccadic eye movements and cognition. Trends in Cognitive Sciences, 4:6-14, 02000.

[27] B. Logan, J. Healey, M. Philipose, E. Tapia, and S. Intille. A Long-Term Evaluation of Sensing Modalities for Activity Recognition. In Proc. of the 9th International Conference on Ubiquitous Computing (UbiComp 2007), pages 483-500. ACM Press, 2007.

[28] P. Lukowicz, H. Junker, M. Stäger, T. von Büren, and G. Tröster. WearNET: A Distributed Multi-sensor System for Context Aware Wearables. In Proc. of the 4th International Conference on Ubiquitous Computing (UbiComp 2002), pages 361-370. Springer, 2002.

[29] H. Manabe and M. Fukumoto. Full-time wearable headphonetype gaze detector. In Ext. Abstracts of the SIGCHI Conference on Human Factors in Computing Systems (CHI 2006), pages 1073-1078. ACM Press, 2006

[30] S. Mann. Wearable Computing as Means for Personal Empowerment. In Proc. of the 1st International Conference on Wearable Computing (ICWC 1999), 1998.

[31] D. Melcher and E. Kowler. Visual scene memory and the guidance of saccadic eye movements. Vision Research, 41(2526):3597-3611, 2001.

[32] S. Mitra and T. Acharya. Gesture Recognition: A Survey. IEEE Transactions on Systems, Man, and Cybernetics, Part C: Applications and Reviews, 37(3):311-324, May 2007.
[33] F. Mizuno, T. Hayasaka, K. Tsubota, S. Wada, and T. Yamaguchi. Development of hands-free operation interface for wearable computer-hyper hospital at home. In Proc. of the 25th Annual International Conference of the Engineering in Medicine and Biology Society (EMBS 2003), volume 4, pages 3740-3743, September 17-21, 2003.

[34] J. B. Pelz, M. M. Hayhoe, and R. Loeber. The coordination of eye, head, and hand movements in a natural task. Experimental Brain Research, 139(3):266-277, aug 2001.

[35] G. R. Philips, A. A. Catellier, S. F. Barrett, and C. H. Wright. Electrooculogram wheelchair control. In Biomedical Sciences Instrumentation, volume 43, pages 164-169, 2007.

[36] P. Qvarfordt and S. Zhai. Conversing with the user based on eye-gaze patterns. In Proc. of the SIGCHI Conference on $\mathrm{Hu}$ man Factors in Computing Systems (CHI 2005), pages 221230. ACM Press, 2005

[37] D. Roggen, B. Arnrich, and G. Tröster. Life Style Management using Wearable Computer. In Proc. of the 4th International Workshop on Ubiquitous Computing for Pervasive Healthcare Applications (UbiHealth 2006), 2006.

[38] G. Ruffini, S. Dunne, E. Farres, P. C. P. Watts, E. Mendoza, S. R. P. Silva, C. Grau, J. Marco-Pallares, L. Fuentemilla, and B. Vandecasteele. ENOBIO - First Tests of a Dry Electrophysiology Electrode using Carbon Nanotubes. In Proc. of the 28th Annual International Conference of the Engineering in Medicine and Biology Society (EMBS 2006), pages 18261829. IEEE, August 30-September 2006, 2006.

[39] G. Schindler, C. Metzger, and T. Starner. A Wearable Interface for Topological Mapping and Localization in Indoor Environments. In Proc. of the 2nd International Workshop on Location- and Context-Awareness, pages 64-73. Springer, 2006.

[40] SensoMotoric Instruments (SMI). iView X HED. http: //www.smivision. com/, 2009.

[41] M. A. Tinati and B. Mozaffary. A Wavelet Packets Approach to Electrocardiograph Baseline Drift Cancellation. International Journal of Biomedical Imaging, Article ID 97157:Article ID 97157, 9 pages, 2006.

[42] A. T. Vehkaoja, J. A. Verho, M. M. Puurtinen, N. M. Nojd, J. O. Lekkala, and J. A. Hyttinen. Wireless Head Cap for EOG and Facial EMG Measurements. In Proc. of the 27th Annual International Conference of the Engineering in Medicine and Biology Society (EMBS 2005), pages 5865-5868, January 17$18,2005$.

[43] S. Venkataramanan, P. Prabhat, S. R. Choudhury, H. B. Nemade, and J. S. Sahambi. Biomedical instrumentation based on electrooculogram (EOG) signal processing and application to a hospital alarm system. In Proc. of the 3rd International Conference on Intelligent Sensing and Information Processing (ICISIP 2005), pages 535-540, January 4-7, 2005.

[44] J. G. Webster. Medical Instrumentation: Application and Design. John Wiley and Sons, New York, 4th edition, 1998.

[45] W. Sardha Wijesoma, Kang S. Wee, Ong C. Wee, A. Prabhath Balasuriya, Koh T. San, and Kow K. Soon. EOG based control of mobile assistive platforms for the severely disabled. In Proc. of the IEEE International Conference on Robotics and Biomimetics (ROBIO 2005), pages 490-494, 2005.

[46] S. Zhai, C. Morimoto, and S. Ihde. Manual and gaze input cascaded (MAGIC) pointing. In Proc. of the SIGCHI Conference on Human Factors in Computing Systems (CHI 1999), pages 246-253. ACM Press, 1999. 